\title{
RESOLUTION AND STABILITY ANALYSIS OF AN INVERSE PROBLEM IN ELECTRICAL IMPEDANCE TOMOGRAPHY - DEPENDENCE ON THE INPUT CURRENT PATTERNS*
}

\author{
DAVID C. DOBSON ${ }^{\dagger}$ AND FADIL SANTOSA ${ }^{\ddagger}$
}

\begin{abstract}
Electrical impedance tomography is a procedure by which one finds the conductivity distribution inside a domain from measurements of voltages and currents at the boundary. This work addresses the issue of stability and resolution limit of such an imaging device. We consider the realistic case where only a finite number of measurements are available. An important feature of our approach, which is based on linearization, is that we do not discretize the unknown conductivity distribution. Instead, we define a pseudo-solution based on least-squares. A goal of this investigation is to compare the stability and resolution power of a system that uses dipole sources, with another that uses trigonometric sources. Our findings are illustrated in numerical calculations.
\end{abstract}

Key words. elliptic inverse problem, electrical impedance tomography, conductivity imaging, stability analysis, resolution limit, conditioning, sensitivity analysis

AMS(MOS) subject classifications. 35R30, 15A09, 65N99

1. Introduction. In electrical impedance tomography, the problem is to find a conductivity distribution in a domain from electrostatic measurements collected at the boundary. The conductivity distribution, when displayed on a gray-level plot is often called an "image", and can be used for diagnostic purposes in certain medical applications. See for instance [4].

Let us consider a two dimensional model problem. For simplicity, we assume that our domain $\Omega$ is the unit disk. The problem is to find the conductivity distribution in the disk from measurements collected on the circle. In order to make the required measurements, $n$ electrodes are attached to the boundary of the domain. The data consist of voltage potentials at the electrodes; the voltage potentials are generated by applying input currents at the electrodes.

Let us denote the input current passing through the electrode $i$ by $f_{i}$. The collection of input currents at the $n$ electrodes is refered to as the "current pattern", and is denoted by the $n$-vector $f$,

$$
f=\left(f_{1}, f_{2}, \cdots, f_{n}\right)^{T} .
$$

A current pattern must satisfy $\sum_{i=1}^{n} f_{i}=0$.

Since the voltage potential is unique up to a constant, we take our data to be voltage drops across adjacent electrodes. That is, let $u_{i}$ be the voltage potential at electrode $i$, then the data, corresponding to a current pattern $f$, is the $\mathbb{R}^{n}$ vector

$$
g=\left(g_{1}, g_{2}, \cdots, g_{n}\right)^{T},
$$

where $g_{i}=u_{i+1}-u_{i}$ (and electrode $n+1$ is identified with electrode 1 ).

A single experiment consists of generating a current pattern $f$ and collecting the corresponding voltage drops $g$. In a typical problem, the measurements consist of $m$

\footnotetext{
* This work is supported by the National Science Foundation and the Air Force Office of Scientific Research. This work was carried out while the first author was at the Institute for Mathematics and its Applications, University of Minnesota.

$\dagger$ School of Mathematics, University of Minnesota, Minneapolis, MN 55455-0436 dobson@ima. umn. edu

$\ddagger$ Department of Mathematical Sciences, University of Delaware, Newark, DE 19716 santosa@math.udel.edu
} 
experiments involving current patterns

$$
f^{(1)}, f^{(2)}, \cdots, f^{(m)},
$$

and $m$ corresponding voltage difference vectors

$$
g^{(1)}, g^{(2)}, \cdots, g^{(m)} .
$$

The inverse problem of electrical impedance tomography is to determine the conductivity distribution inside the disk, given the currents $f^{(j)}$ and the measurements $g^{(j)}$, $j=1, \cdots, m$. The total number of data points is thus $n \times m$.

A mathematical model for this problem is as follows. We use polar coordinates $(r, \theta)$; hence our domain $\Omega$ is $\{r<1\}$. Let $u$ denote the voltage potential corresponding to the current pattern $f$. The function $u$ satisfies

$$
\nabla \cdot(\sigma \nabla u)=0 \text { for } r<1
$$

where $\sigma(r, \theta)$ is the conductivity of the medium. The electrodes are of width $h$ and their centers are at coordinates $\left(1, \theta_{i}\right), \theta_{i}=2 \pi(i-1) / n$. We model the input currents at the electrodes with the Neumann boundary condition

$$
\left(\sigma \frac{\partial u}{\partial r}\right)(1, \theta)=\sum_{i=1}^{n} f_{i} \chi\left(\theta, \theta_{i}\right)
$$

where $\chi$ is the characteristic function

$$
\chi\left(\theta, \theta_{i}\right)=\left\{\begin{array}{lll}
1 / h & \text { for } & \left|\theta-\theta_{i}\right| \leq h / 2 \\
0 & \text { otherwise }
\end{array} .\right.
$$

The data for this experiment consist of voltage drops

$$
u\left(1, \theta_{i+1}\right)-u\left(1, \theta_{i}\right)=g_{i}, \quad \text { for } \quad i=1, \cdots, n .
$$

Since $\sum_{i=1}^{n} f_{i} \chi\left(x, x_{i}\right) \in L^{2}(\partial \Omega)$, problem (1) with the normalization $\int_{0}^{2 \pi} u(1, \theta) d \theta=0$ and the boundary condition (1b) is a well-posed boundary value problem for $u$. For other electrode models, see [7].

In order to state the inverse problem, let us denote by $F(\sigma ; f)$ the map that takes a given conductivity distribution, for a given prescribed current pattern $f$, to the voltage drops across the adjacent electrodes. Thus $F(\cdot ; f)$ takes values in $\mathbb{I}^{n}$; the $i$-th component of this map is

$$
(F(\sigma ; f))_{i}:=u\left(1, \theta_{i+1}\right)-u\left(1, \theta_{i}\right)
$$

where it is understood that $u$ solves (1a), (1b).

In our problem, we choose $m$ current patterns $f^{(j)}$, and measure the corresponding voltage drops, $g^{(j)}, j=1, \cdots, m$. Then, the inverse problem is to determine, to the extent possible, the function $\sigma$ such that

$$
F\left(\sigma, f^{(j)}\right)=g^{(j)}, \quad j=1, \cdots, m
$$

Clearly, $\sigma$ is not completely determined by the data. A quick parameter count indicates that we have $n \times m$ pieces of real data from which we are to find a function, which, in principle, has an infinite number of unknowns. Moreover, as we shall see 
later, whatever is determinable from the measurement set may be unstable in the sense that small errors in the data could lead to large errors in the "solution".

In this work, we will study two measurement systems; one uses so-called "dipole" current patterns [2], [3], and the other, "trigonometric" current patterns. Isaacson et al [9], [11] have shown that adaptive current patterns (adapted to the unknown conductivity) are desirable. Trigonometric current patterns are optimal in the sense of Isaacson when the unknown conductivities are radially symmetric. We remark that all existing electrical impedance tomography systems we know of presently use either dipole or trigonometric current patterns for imaging.

Our work attempts to address the following issues:

- Characterize the part of the conductivity (image) that is determinable from a data set for a given noise level,

- Identify the part of the image that is lost due to the presence of noise in the data,

- Assess the stability of the image reconstruction.

One goal of this work is to quantify the information content of the data for a given measurement system.

Our previous work [1] [8] addressed some of the same issues for the theoretical case where there are an infinite number of electrodes. Cheney and Isaacson [6] investigated the effects of measurement errors, finite number of electrodes, and modeling errors on the reconstruction of the image. Their work lead to estimates of the errors in the Fourier transform of the image.

The method outlined in this paper is quite general, and with a little computation, leads to a quantitative characterization of the properties of the inverse problem. In order to facilitate our investigation, we shall consider the linearized inverse problem. The linearization is justified if the conductivity $\sigma$ is a small perturbation from a constant. The properties of the linearized problem provide a useful picture of the behavior of the fully nonlinear problem. Understanding the properties of the linearized problem is also valuable in designing Newton-type algorithms, where at each iteration, one must solve a linearized problem.

2. The linearized inverse problem. To linearize the problem, we assume that the conductivity $\sigma(r, \theta)$ is of the form

$$
\sigma(r, \theta)=1+\delta \sigma(r, \theta),
$$

where $\delta \sigma$ is small (say in $L^{\infty}(\Omega)$-norm). In addition, we make the assumption that the support of $\delta \sigma$ is contained in $\Omega^{\prime}$ where $\Omega^{\prime} \subset \subset \Omega$ (for convenience, let $\Omega^{\prime}=\{(r, \theta) \mid r<$ $\left.\left.r^{\prime}<1\right\}\right)$. We now assume that a voltage potential $u(r, \theta)$ satisfying (1) can be written in the form

$$
u(r, \theta)=U(r, \theta)+\delta u(r, \theta),
$$

where $\delta u$ is small in comparison to $U$ in some norm. Considering $\delta \sigma$ and $\delta u$ as perturbations, we insert (3) and (4) in (1a) and (1b). After dropping terms involving products of perturbations and collecting terms of equal orders of magnitude, we find that the background field $U(r, \theta)$ satisfies

$$
\begin{aligned}
& \triangle U=0 \text { for for } r<1, \\
& \frac{\partial U}{\partial r}(1, \theta)=\sum_{i=1}^{n} f_{i} \chi\left(\theta, \theta_{i}\right)
\end{aligned}
$$


where $\chi\left(\theta, \theta_{i}\right)$ is the characteristic function defined in (2). We also require $U$ to satisfy the normalization condition $\int_{0}^{2 \pi} U(1, \theta) d \theta=0$. The perturbational field $\delta u(r, \theta)$ satisfies

$$
\begin{gathered}
\triangle \delta u=-\nabla \cdot \delta \sigma \nabla U \text { for } r<1, \\
\frac{\partial \delta u}{\partial r}(1, \theta)=0,
\end{gathered}
$$

where we again enforce the normalization $\int_{0}^{2 \pi} \delta u(1, \theta) d \theta=0$. We emphasize that $U$ depends on the current pattern $f$. Note also that because of this dependence, $\delta u$ is also dependent on $f$.

The linearized forward map will be denoted by $D F$. The linearized map $D F$ takes a conductivity perturbation $\delta \sigma$, for a given a current pattern $f$, to perturbational voltage drops on the boundary, $r=1$. The $i$-th component of this map is

$$
(D F(f) \delta \sigma)_{i}:=\delta u\left(1, \theta_{i+1}\right)-\delta u\left(1, \theta_{i}\right) .
$$

We view the map as

$$
D F(f): L^{2}\left(\Omega^{\prime}\right) \rightarrow \mathbb{R}^{n} .
$$

In this notation, we have explicitly stated parametric dependence of the map on the current pattern $f$. The dependence is through the background field $U(x)$, and is therefore linear.

Notice that by elliptic regularity, the background field $U$ is smooth and bounded in $\Omega^{\prime}$, so that the right-hand side of $(6 \mathrm{a})$ is a distribution in $H^{-1}(\Omega)$. It follows that the solution $\delta u$ to problem $(6 \mathrm{a})-(6 \mathrm{~b})$ is in $H^{1}(\Omega)$. We then have that $\left.\delta u\right|_{\partial \Omega^{\prime}} \in H^{1 / 2}\left(\partial \Omega^{\prime}\right)$ and since $\delta u$ is harmonic in the region $\Omega-\Omega^{\prime}$, it must be smooth and hence continuous on the outer boundary $\partial \Omega$. Thus $\delta u$ is well-defined pointwise on $\partial \Omega$ and (7) makes sense. So the map $D F$ is well-defined over $L^{2}\left(\Omega^{\prime}\right)$.

In the linearized inverse problem, the goal is to find $\delta \sigma$ from knowledge of the differences between the measured voltage drops and the background voltage drops $U\left(1, \theta_{i+1}\right)-U\left(1, \theta_{i}\right)$ for a set of current patterns $f^{(j)}, j=1, \cdots, m$. Let us denote the background voltage due to current pattern $f^{(j)}$ by $U^{(j)}(x)$. The linearized inverse problem is to determine $\delta \sigma$ in the equation

$$
D F\left(f^{(j)}\right) \delta \sigma=g^{(j)}-T U^{(j)}=: \delta g^{(j)}, \quad \text { for } \quad j=1, \cdots, m,
$$

where $T U^{(j)}$ is the $I R^{n}$-vector whose components are $\left(U^{(j)}\left(1, \theta_{i+1}\right)-U^{(j)}\left(1, \theta_{i}\right)\right)$. Again, $\delta \sigma$ is clearly not determined because of lack of data. Note that it is also apparent that whatever part of $\delta \sigma$ we can determine from the finite data set will be dependent on the choice of current patterns $f^{(j)}$.

We can state the linearized problem more succinctly as a "moment problem" by explicitly obtaining a formula for the linearized forward map. This is facilitated by the Calderon identity [5]

$$
-\int_{0}^{2 \pi} \delta u \frac{\partial v}{\partial r}(1, \theta) d \theta=\int_{0}^{2 \pi} \int_{0}^{1} \delta \sigma(r, \theta) \nabla U \cdot \nabla v r d r d \theta,
$$

which is valid for a harmonic $v$. To obtain the $i$-th component of the map $(D F(f) \delta \sigma)_{i}$, we choose $v^{(i)}(r, \theta)$ be the generalized solution to

$$
\triangle v^{(i)}=0 \text { in } \Omega
$$




$$
\frac{\partial v^{(i)}}{\partial r}(1, \theta)=\delta\left(\theta-\theta_{i}\right)-\delta\left(\theta-\theta_{i+1}\right) .
$$

Inserting this in (10), we get

$$
\delta u\left(1, \theta_{i+1}\right)-\delta u\left(1, \theta_{i}\right)=\int_{0}^{2 \pi} \int_{0}^{1} \delta \sigma(r, \theta) \nabla U \cdot \nabla v^{(i)} r d r d \theta .
$$

Using our assumption that $\delta \sigma$ is supported in $\Omega^{\prime}$, the linearized forward map is

$$
(D F(f) \cdot \delta \sigma)_{i}=\int_{0}^{2 \pi} \int_{0}^{r^{\prime}} \delta \sigma(r, \theta) \nabla U \cdot \nabla v^{(i)} r d r d \theta .
$$

Thus, the linearized inverse problem is to find $\delta \sigma(r, \theta)$ such that

$$
\int_{0}^{2 \pi} \int_{0}^{r^{\prime}} \delta \sigma \nabla U^{(j)} \cdot \nabla v^{(i)} r d r d \theta=\delta g_{i}^{(j)} \quad \text { for } \quad i=1, \cdots, n, \quad j=1, \cdots, m .
$$

This problem takes the form of a moment problem - we are given integrals of the desired function $\delta \sigma(r, \theta)$ against weights of the form $\nabla U^{(j)} \cdot \nabla v^{(i)}$.

3. Pseudo-inverse and information content of the data. At this point, one could discretize the unknown $\delta \sigma$ with $p$ parameters, and view the linearized forward map as a map from $\mathbb{R}^{p}$ to $\mathbb{R}^{n}$. The linearized inverse problem with $m$ current patterns amounts to finding the solution of a linear system with $n \times m$ equations and $p$ unknowns. We could apply a standard technique, such as singular value decomposition (SVD), to study the stability and resolvability of the problem. Such a study would certainly lead to useful information about the problem. Unfortunately, the information will be dependent on the discretization of the unknown conductivity $\delta \sigma$. To avoid this undesirable property, we will analyze the problem without relying on discretization.

To this end, we must replace the SVD with a counterpart which makes sense for linear maps from $L^{2}\left(\Omega^{\prime}\right)$ to $\mathbb{R}^{m n}$. For clarity, consider the linearized inverse problem with one current pattern $f$ : Find $\delta \sigma$ such that

$$
(D F(f) \cdot \delta \sigma)_{i}=\int_{0}^{2 \pi} \int_{0}^{r^{\prime}} \delta \sigma(r, \theta) \nabla U \cdot \nabla v^{(i)} r d r d \theta=\delta g_{i} \quad \text { for } \quad i=1, \cdots, n \text {. }
$$

Assume that the data $\delta g$ is in the range of linearized map. Then we call $\delta \sigma^{+}$a pseudo-solution of the linearized problem if $\delta \sigma^{+}$is the minimizer of the constrained problem

$$
\min _{\operatorname{subj} D F(f) \delta \sigma=\delta g}\|\delta \sigma\|_{L^{2}\left(\Omega^{\prime}\right)}^{2} .
$$

This definition of the pseudo-inverse is similar to the conditions for the generalized inverse or the Moore-Penrose inverse in matrix calculations [10]. The only notable difference being that we assume a priori that $\delta g$ is in the range of $D F(f)$. We argue that this solution is a "reasonable" choice by noting that given the finite amount of data, we expect to have many $\delta \sigma$ which would satisfy equation (13). In the absence of additional information on $\delta \sigma$, we choose, among all $\delta \sigma$ 's that fit the data, one which has the least $L^{2}$-norm. We remark that an alternative approach for solving the moment problem (13) and estimating the information content of the data is given by 
the Backus-Gilbert method. See [12] for a discussion of the Backus-Gilbert method and its (close) relation to the pseudo-inverse solution.

We can solve (14) by using the Lagrange multiplier method. That is, we consider the minimization of the functional

$$
J(\delta \sigma, \lambda)=\int_{0}^{r^{\prime}} \int_{0}^{2 \pi}|\delta \sigma(r, \theta)|^{2} r d r d \theta+\sum_{i=1}^{n} \lambda_{i}\left(\int_{0}^{r^{\prime}} \int_{0}^{2 \pi} \delta \sigma(r, \theta) \nabla U \cdot \nabla v^{(i)} r d r d \theta-\delta g_{i}\right) .
$$

A necessary condition for the minimum is that

$$
\delta \sigma^{+}=\left\{\begin{array}{ccc}
\sum_{i=1}^{n} \phi_{i} \nabla U \cdot \nabla v^{(i)} & \text { for } & r \leq r^{\prime} \\
0 & \text { otherwise }
\end{array}\right.
$$

for some $\phi \in \mathbb{R}^{n}$.

The representation (15) can be intepreted as a statement about the information content of one experiment with the current pattern $f$. It states that based on the pseudo-inverse criterion we used, the part of $\delta \sigma$ that can be determined from the data $\delta g$ must be of the form given by (15). The representation is explicit since both $U$ and $v^{(i)}$ are known; recall that $U(r, \theta)$ is the background voltage potential (dependent on $f)$, and $v^{(i)}(r, \theta)$ solves (11a-11b). The resolvable conductivity $\delta \sigma^{+}(r, \theta)$ is at most $n$ dimensional; the number $n$ corresponds to the $n$ voltage drops at the electrodes.

Given the form that $\delta \sigma^{+}$must take, we can determine it from the data $\delta g$ by inserting the representation (15) in equation (13) and solving for $\phi$. Before we address this issue further, let us derive the representation for the case where $m$ current patterns have been used.

Following the previous notation, let the data for current pattern $f^{(j)}$ be $\delta g^{(j)}$. We perform $m$ experiments so that $j=1, \cdots, m$. The linearized inverse problem is to find $\delta \sigma(x)$ which satisfies (12). Again, we assume that the data set $\delta g^{(j)}$ for $j=1, \cdots, m$ is in the range of $D F\left(f^{(j)}\right)$. From the representation for the case of one experiment, it is apparent that the pseudo-inverse for the case of $m$ experiments must take the form of

$$
\delta \sigma^{+}=\left\{\begin{array}{ccc}
\sum_{j=1}^{m} \sum_{i=1}^{n} \phi_{i}^{(j)} \nabla U^{(j)} \cdot \nabla v^{(i)} & \text { for } & r \leq r^{\prime} \\
0 & \text { otherwise }
\end{array},\right.
$$

for some vectors $\phi^{(j)}$. The number of degrees of freedom is $m \times n$, and corresponds to the fact that for each current pattern, we collect $n$ real data; a total of $m$ current patterns have been used.

The representation (16) can be interpreted as the conductivity image that is resolvable from the data for a particular experimental setup. Given a conductivity distribution $\delta \sigma(r, \theta)$, and $m$ current patterns, $f^{(j)}, j=1, \cdots, m$, we can find out just how much of the original $\delta \sigma(r, \theta)$ is "visible" from this set of measurements. To do this, we project the true image $\delta \sigma(r, \theta)$ onto the subspace whose elements are of the form $\delta \sigma^{+}$. Let

$$
V=\operatorname{span}\left\{\nabla U^{(j)} \cdot \nabla v^{(i)} ; i=1, \cdots, n, j=1, \cdots, m\right\} .
$$

The decomposition above helps us to describe the visible part of the conductivity image. Let $\delta \sigma$ be a conductivity perturbation which we want to determine. The part of $\delta \sigma$ that is visible by the experiment is the projection of $\delta \sigma$ onto $V$. Such a calculation can be used to quantify the limitations of an experimental setup. We note 
that the elements $\nabla U^{(j)} \cdot \nabla v^{(i)}$ may be linearly dependent, and hence the dimension of $V$ may be less than the number of elements, $m n$.

While the analysis above describes the information content of a data set, that is, it characterizes what part of an image $\delta \sigma$ can be reconstructed from the data, it does not provide any information about how well we can find the determinable part of $\delta \sigma(r, \theta)$ when the data are contaminated by measurement and modelling error. This is the issue of stability, which we address next.

4. Stability. Given that we can at best, for a fixed experimental setup, recover a conductivity distribution that is of the form (16), we still need to determine it. This is easily done by inserting (16) back into the functional equation for the linearized inverse problem (12). We find that $\phi^{(j)}$ must solve

$$
\begin{gathered}
\int_{0}^{r^{\prime}} \int_{0}^{2 \pi} \sum_{l=1}^{m} \sum_{k=1}^{n} \phi_{k}^{(l)}\left(\nabla U^{(j)} \cdot \nabla v^{(i)}\right)\left(\nabla U^{(l)} \cdot \nabla v^{(k)}\right) r d r d \theta=\delta g_{i}^{(j)} \\
\text { for } \quad i=1, \cdots, n, \quad j=1, \cdots, m .
\end{gathered}
$$

Let us write this equation in matrix form. Define $K^{(j, l)}$ to be the $n \times n$ matrix whose elements are

$$
K_{i k}^{(j, l)}=\int_{0}^{r^{\prime}} \int_{0}^{2 \pi}\left(\nabla U^{(j)} \cdot \nabla v^{(i)}\right)\left(\nabla U^{(l)} \cdot \nabla v^{(k)}\right) r d r d \theta
$$

Each term $\phi^{(l)}$ is an $\mathbb{R}^{n}$-vector. Hence, we can rewrite (17) in the form

$$
\left[\begin{array}{cccc}
K^{(1,1)} & K^{(1,2)} & \cdots & K^{(1, m)} \\
K^{(2,1)} & K^{(2,2)} & \cdots & K^{(2, m)} \\
\cdots & \cdots & & \cdots \\
\cdots & \cdots & & \cdots \\
K^{(m, 1)} & K^{(m, 2)} & \cdots & K^{(m, m)}
\end{array}\right]\left[\begin{array}{c}
\phi^{(1)} \\
\phi^{(2)} \\
\cdot \\
\cdot \\
\phi^{(m)}
\end{array}\right]=\left[\begin{array}{c}
\delta g^{(1)} \\
\delta g^{(2)} \\
\cdot \\
\cdot \\
\delta g^{(m)}
\end{array}\right] .
$$

The linearized inverse problem, in the context of the pseudo-solution that we defined, has now been reduced to the problem of solving the linear system (19) for $m$ vectors $\phi^{(l)} \in \mathbb{R}^{n}$.

Denote the matrix on the left-hand side of (19) by $\mathbf{K} ; \mathbf{K}$ is an $m n \times m n$ square matrix. Each subblock of this matrix is

$$
K^{(j l)}=D F\left(f^{(j)}\right) D F\left(f^{(l)}\right)^{T} .
$$

The elements of $\mathbf{K}$ possess the symmetry

$$
K_{i k}^{(j l)}=K_{k i}^{(l j)},
$$

as can be seen in (18). The first row of the submatrix $K^{(j l)}$ is

$$
\left(K_{11}^{(j l)}, K_{12}^{(j l)}, \cdots, K_{1 n}^{(j l)}\right) .
$$

Compare this with the first column of the submatrix $K^{(l j)}$, which is

$$
\left(K_{11}^{(l j)}, K_{21}^{(l j)}, \cdots, K_{n 1}^{(l j)}\right)^{T} .
$$

The symmetry of the elements $(20)$ lead us to conclude that first row of $K^{(j l)}$ is the same as the first column of $K^{(l j)}$. The same conclusion holds for the other rows of $K^{(j l)}$ and the other columns of $K^{(l j)}$. Therefore, the matrix $\mathbf{K}$ is symmetric. 
At this point, note that we do not expect the matrix $\mathbf{K}$ to be invertible in general because there is no guarantee that the columns of $\mathbf{K}$ are independent. We argue that an acceptable solution is the pseudo-inverse solution of the linear system.

Since $\mathbf{K}$ is symmetric, we can diagonalize (SVD) $\mathbf{K}$ into

$$
\mathbf{K}=\mathbf{Q} \mathbf{\Sigma} \mathbf{Q}^{T}
$$

where $\mathbf{Q}$ is an orthonormal matrix, and $\boldsymbol{\Sigma}$ is a diagonal matrix of eigenvalues of $\mathbf{K}$. Let $\Phi$ and $\Psi$ be two columns of $\mathbf{Q}$ whose eigenvalues are $\lambda$ and $\nu$, respectively. We rewrite $\Phi$ in the form

$$
\Phi=\left[\phi^{(1)^{T}}, \phi^{(2)^{T}}, \cdots, \phi^{(m)^{T}}\right]^{T},
$$

where each $\phi^{(j)}$ is an $\mathbb{R}^{n}$ vector. The conductivity image associated with $\Phi$ is

$$
\delta \sigma_{\Phi}=\sum_{i=1}^{n} \sum_{j=1}^{m} \phi_{i}^{(j)} \nabla U^{(j)} \cdot \nabla v^{(i)} .
$$

Similarly, the image associated with the vector $\Psi$ is

$$
\delta \sigma_{\Psi}=\sum_{i=1}^{n} \sum_{j=1}^{m} \psi_{i}^{(j)} \nabla U^{(j)} \cdot \nabla v^{(i)},
$$

where we have used the same decomposition on $\Psi$ as on $\Phi$. Next, we compute the inner product of $\delta \sigma_{\Phi}$ with $\delta \sigma_{\Psi}$,

$$
\begin{aligned}
& \int_{0}^{2 \pi} \int_{0}^{r^{\prime}} \delta \sigma_{\Phi} \delta \sigma_{\Psi} r d r d \theta \\
& =\sum_{i=1}^{n} \sum_{j=1}^{m} \sum_{k=1}^{m} \sum_{l=1}^{m} \phi_{i}^{(j)} \psi_{k}^{(l)} \int_{0}^{2 \pi} \int_{0}^{r^{\prime}}\left(\nabla U^{(j)} \cdot \nabla v^{(i)}\right)\left(\nabla U^{(k)} \cdot \nabla v^{(k)}\right) r d r d \theta \\
& =\Phi^{T} \mathbf{K} \Psi \\
& =\nu \Phi^{T} \Psi \\
& =0
\end{aligned}
$$

Therefore, associated with the columns of $\mathrm{Q}$ is an orthogonal set of conductivity perturbations.

The pseudo-inverse solution to the linear system (19) is a linear combination of the columns of $\mathbf{Q}$. This means that the conductivity distribution defined by the pseudo-inverse is a linear combination of the orthogonal conductivities associated with the columns of $\mathbf{Q}$. Since the columns of $\mathbf{Q}$ are orthogonal, the pseudo-inverse solution of (19) for $\phi^{(j)}$ corresponds to the pseudo-inverse solution in the conductivity perturbation $\delta \sigma$. In other words, by finding the pseudo-inverse of (19) and inserting it in (16), we get a conductivity image that is consistent with the data and has minimal $L^{2}\left(\Omega^{\prime}\right)$-norm.

The stability of the solution of (19) is determined by the singular values (eigenvalues) of the matrix $\mathbf{K}$. Suppose the largest singular value of $\mathbf{K}$ is $\lambda_{1}$ and the smallest nonzero singular value is $\lambda_{p}$ for some $p \leq m n$. The condition number of the matrix, in the context of the pseudo-inverse, is

$$
\kappa=\frac{\lambda_{1}}{\lambda_{p}}
$$


This number tells us how stably we can reconstruct the resolvable image from the data. The number $\kappa$ can be viewed as the error magnification factor. As we shall see, the problem can be very ill-conditioned.

A better way to use the singular value decomposition in solving ill-conditioned problem as follows. Suppose that we will only tolerate error magnification of some fixed value $M$. Then we construct the pseudo-inverse by including singular vectors whose singular values are larger or equal to $\lambda_{1} / M$. See Golub and van Loan [10]. Such a construction guarantees that the condition number of the pseudo-inverse will be approximately equal to, but no greater than, $M$.

In the presence of uncertainty in data, we see that stability itself further restricts the level of resolution of a conductivity image. To properly assess the resolution limit of a measurement device, we must find out what part of an image $\delta \sigma$ is recoverable from a data set containing errors.

An experiment is characterized by

- Current patterns $f^{(j)}, j=1, \cdots, m$,

- Condition number $M$ of the pseudo-inverse (error magnification tolerance), based on a priori estimate of measurement errors.

To assess what part of a given image $\delta \sigma(r, \theta)$ can be "seen" by such a system, consider the linear system (19) using

$$
\left[\left(D F\left(f^{(1)}\right) \cdot \delta \sigma\right)^{T},\left(D F\left(f^{(2)}\right) \cdot \delta \sigma\right)^{T}, \cdots,\left(D F\left(f^{(m)}\right) \cdot \delta \sigma\right)^{T}\right]^{T}
$$

as the right-hand side, and find the pseudo-inverse which includes singular vectors whose singular values are greater than or equal to $\lambda_{1} / M$. Denote the solution by $\phi^{(j)+}$. The resulting image, given by

$$
\delta \sigma^{+}=\left\{\begin{array}{ccc}
\sum_{j=1}^{m} \sum_{i=1}^{n} \phi^{(j)}+\left(\nabla U^{(j)} \cdot \nabla v^{(i)}\right) & \text { for } & r \leq r^{\prime} \\
0 & \text { otherwise }
\end{array},\right.
$$

is what is resolvable by the system for the given condition number $M$.

5. Relationship among current patterns. For the dipole current pattern, in the $j$-th experiment, we have current flowing into electrode $j$ and out of electrode $j+1$. We use $d^{(j)}$ to denote this pattern, which is given by

$$
d^{(j)}=[0,0, \cdots, 0,1,-1,0, \cdots]^{T},
$$

where the entry 1 appears as the $j$-th entry. The dipole pattern $d^{(1)}$ is

$$
d^{(1)}=[1,-1,0, \cdots, 0]^{T}
$$

the other dipole patterns are simply rotations of this vector.

Recall that current patterns $f$ must satisfy $\sum_{i=1}^{n} f_{i}=0$. Any current pattern is thus a linear combination of the dipole patterns, and hence the linearized forward map corresponding to any current pattern can be extracted from the linearized forward maps corresponding to the full set of dipole patterns.

Let $f$ be an $\mathbb{R}^{n}$ vector corresponding to some current pattern. To find a representation for $f$ in terms of a linear combination of the dipole patterns, we solve

$$
\left[\begin{array}{rrrrrr}
1 & 0 & . & . & . & -1 \\
-1 & 1 & . & . & . & 0 \\
0 & -1 & . & . & . & 0 \\
. & 0 & . & . & . & 0 \\
. & . & . & . & . & \cdot \\
0 & 0 & . & . & . & 1
\end{array}\right]\left[\begin{array}{r}
x_{1} \\
x_{2} \\
x_{3} \\
\cdot \\
\cdot \\
x_{n}
\end{array}\right]=\left[\begin{array}{r}
f_{1} \\
f_{2} \\
f_{3} \\
\cdot \\
\cdot \\
f_{n}
\end{array}\right]
$$


The columns of the matrix above are the dipole patterns; the location of the 1 entry reveals the location of the input dipole source. The coefficients $x_{j}, j=1, \cdots, n$ is the weight in the linear combination

$$
f=\sum_{j=1}^{n} x_{j} d^{(j)}
$$

The background potential corresponding to the current pattern $f$ is then

$$
U(r, \theta)=\sum_{j=1}^{n} x_{j} V^{(j)}(r, \theta)
$$

where $V^{(j)}$ is the background field due to dipole $d^{(j)}$. Therefore, the linearized forward map corresponding to the current pattern $f$ is related to those of the dipole current patterns by

$$
D F(f) \delta \sigma=\sum_{j=1}^{n} x_{j} D F\left(d^{(j)}\right) \delta \sigma .
$$

When $m$ experiments are carried out with current patterns $f^{(j)}$ for $j=1, \cdots, m$, the resolvable image is (16),

$$
\delta \sigma^{+}=\left\{\begin{array}{cc}
\sum_{j=1}^{m} \sum_{i=1}^{n} \phi_{i}^{(j)} \sum_{j^{\prime}=1}^{n} x_{j^{\prime}}^{(j)} \nabla V^{\left(j^{\prime}\right)} \cdot \nabla v^{(i)} & \text { for } \\
0 & \text { otherwise }
\end{array}\right.
$$

In the preceeding equation, $x^{(j)}$ satisfies

$$
f^{(j)}=\sum_{j^{\prime}=1}^{n} x_{j^{\prime}}^{(j)} d^{\left(j^{\prime}\right)}
$$

From the definition of the matrix element $K_{i k}^{(j, l)}$ in equation (17), it is apparent that if $K_{i k}^{(j, l)}$ is the element corresponding to the dipole patterns, the element corresponding to the set of patterns $f^{(j)}, j=1, \cdots, m$, is

$$
\tilde{K}_{i k}^{(j, l)}=\sum_{j^{\prime}=1}^{n} \sum_{l^{\prime}=1}^{n} x_{j^{\prime}}^{(j)} K_{i k}^{\left(j^{\prime}, l^{\prime}\right)} x_{l^{\prime}}^{(l)}
$$

In matrix form, we can write

$$
\tilde{\mathbf{K}}=\mathbf{X} \mathbf{K} \mathbf{X}^{T}
$$

where

$$
\mathbf{X}=\left[\begin{array}{cccc}
x_{1}^{(1)} I & x_{2}^{(1)} I & \cdots & x_{n}^{(1)} I \\
x_{1}^{(2)} I & x_{2}^{(2)} I & \cdots & x_{n}^{(2)} I \\
\cdots & \cdots & & \cdots \\
\cdots & \cdots & & \cdots \\
x_{1}^{(m)} I & x_{2}^{(m)} I & \cdots & x_{n}^{(m)} I
\end{array}\right]
$$


Here, $I$ is an $n$-by- $n$ identity matrix. The system using dipole current patterns can thus be used as a basis for studying a system using current patterns $f^{(j)}, j=1, \cdots, m$. We solve for $x^{(j)}$ from $f^{(j)}$ and insert them in (23) to obtain $\tilde{\mathbf{K}}$ from $\mathbf{K}$. The resolvable image is found by finding a pseudo-solution of the system (19) with matrix $\mathbf{K}$, and inserting it in (22).

The linear system (21) can be solved in closed form using Discrete Fourier Transforms. Note that the matrix-vector product is merely the discrete convolution of $d^{(1)}$ with the vector $x$. In the Fourier Transform domain, convolutions are products. The Discrete Fourier Transform of a vector $x \in \mathbb{R}^{n}$ is

$$
X_{k}=\sum_{j=1}^{n} x_{j} \exp \left(-\frac{2 \pi i(j-1)(k-1)}{n}\right), \quad \text { for } \quad k=1,2, \cdots, n .
$$

The inversion formula is given by

$$
x_{j}=\frac{1}{n} \sum_{k=1}^{n} X_{k} \exp \left(\frac{2 \pi i(j-1)(k-1)}{n}\right) .
$$

Let $D$ and $F$ stand for the Discrete Fourier Transforms of $d^{(1)}$ and $f$, respectively. Note that $D_{1}=0$. Using the properties of Fourier Transforms on convolutions, we find that

$$
X_{k}= \begin{cases}0 & \text { if } \quad k=1 \\ F_{k} / D_{k} & \text { otherwise }\end{cases}
$$

Once $X_{k}$ is found, it is inserted in the inversion formula to produce $x_{j}$.

We can thus explicitly calculate the matrix $\mathbf{X}$ which transforms the matrix $\mathbf{K}$ corresponding to dipole current patterns into the matrix $\tilde{\mathbf{K}}$ corresponding to trigonometric patterns. The full set of trigonometric current patterns are vectors $f$ with elements

$$
f_{j}= \begin{cases}\cos \left(2 \pi \frac{(j-1)(k-1)}{n}\right) & k=2, \cdots, n / 2+1 \\ \sin \left(2 \pi \frac{(j-1)(k-1)}{n}\right) & k=2, \cdots, n / 2\end{cases}
$$

(for convenience, we assumed that $n$ is even). There are a total of $n-1$ current patterns. By direct calculation, we found that the coefficients in the linear combination to produce the cosines are

$$
x_{j}=\frac{\cos \left(2 \pi \frac{(j-1)(k-1)}{n}\right)-\cos \left(2 \pi \frac{j(k-1)}{n}\right)}{2-2 \cos \left(2 \pi \frac{(k-1)}{n}\right)}, \quad \text { for } \quad j=1, \cdots, n ; \quad k=2, \cdots, n / 2+1,
$$

and for the sines,

$$
x_{j}=\frac{-\sin \left(2 \pi \frac{(j-1)(k-1)}{n}\right)+\sin \left(2 \pi \frac{j(k-1)}{n}\right)}{2-2 \cos \left(2 \pi \frac{(k-1)}{n}\right)}, \quad \text { for } \quad j=1, \cdots, n ; \quad k=2, \cdots, n / 2 .
$$

This calculation gives an explicit formula for the ingredients needed to compute $\tilde{\mathbf{K}}$ from $\mathbf{K}$. 
6. Point electrodes. We consider here the special case where the electrodes are "point" electrodes. This leads to further simplifications which are afforded by the simplicity of the solution for $U^{(j)}$. In the case of point electrodes, we have, instead of (5b), the Neumann condition

$$
\frac{\partial U}{\partial r}(1, \theta)=\sum_{i=1}^{n} f_{i} \delta\left(\theta-\theta_{i}\right) .
$$

As mentioned in the last section, we can use the solutions for the dipoles as a foundation for studying all other current patterns. Therefore, we will study the potential due to a dipole.

In the dipole current pattern, when current is flowing from electrode $j$ to electrode $j+1$, and we have point electrodes, the appropriate boundary condition is

$$
\frac{\partial U}{\partial r}(1, \theta)=\delta\left(\theta-\theta_{j}\right)-\delta\left(\theta-\theta_{j+1}\right) .
$$

This is exactly the same boundary condition as for the harmonic functions $v^{(i)}$, see (11b). Therefore, the background potential $U^{(j)}$ for this dipole pattern (current from $j$ to $j+1$ ) is equal to $v^{(j)}$.

We can compute $v^{(j)}$ using conformal mapping. In complex variable $z=x+\mathbf{i} y$, the active electrodes are located at

$$
z_{j}=\exp \mathbf{i} \theta_{j} \quad \text { and } \quad z_{j+1}=\exp \mathbf{i} \theta_{j+1} .
$$

We find a conformal map $\zeta(z)$ which takes $z_{1}, z_{2}$ and the point $z_{*}=\exp \left(\mathbf{i}\left(\theta_{j}+\theta_{j+1}\right) / 2\right)$ to (complex Cartesian coordinates)

$$
1, \quad-1, \quad \mathbf{i}
$$

This map is the fractional linear transformation given by

$$
\zeta(z)=\frac{a-c z}{d z-b}
$$

where

$$
\begin{aligned}
& a=-(1+\mathbf{i}) z_{j+1}+(1-\mathbf{i}) z_{j} \kappa \\
& b=(1+\mathbf{i}) z_{j+1}+(1-\mathbf{i}) z_{j} \kappa \\
& c=-(1+\mathbf{i})+(1-\mathbf{i}) \kappa \\
& d=(1+\mathbf{i})+(1-\mathbf{i}) \kappa \\
& \kappa=\left(z_{j+1}-z_{*}\right) /\left(z_{j}-z_{*}\right)
\end{aligned}
$$

We compose this map with the complex potential field when the current is flowing from coordinates $(1,0)$ to $(-1,0)$, which is

$$
g(z)=\frac{1}{\pi} \log \frac{1+z}{1-z}
$$

The desired potential field is then

$$
v^{(j)}=\operatorname{Re} g(\zeta(z)) .
$$


The gradient of $v^{(j)}$ is

$$
\nabla v^{(j)}=\frac{1}{\pi}\left(\operatorname{Re} \frac{d}{d z} g(\zeta(z)),-\operatorname{Im} \frac{d}{d z} g(\zeta(z))\right) .
$$

The matrix $\mathbf{K}$ for the dipole patterns then is calculated using [cf. (18)]

$$
K_{i k}^{(j, l)}=\int_{0}^{r^{\prime}} \int_{0}^{2 \pi}\left(\nabla v^{(j)} \cdot \nabla v^{(i)}\right)\left(\nabla v^{(l)} \cdot \nabla v^{(k)}\right) r d r d \theta .
$$

The representation for the determinable part of the image is (16)

$$
\delta \sigma^{+}=\left\{\begin{array}{ccc}
\sum_{j=1}^{m} \sum_{i=1}^{n} \phi_{i}^{(j)} \nabla v^{(j)} \cdot \nabla v^{(i)} & \text { for } & r \leq r^{\prime} \\
0 & \text { otherwise. }
\end{array}\right.
$$

Notice that due to symmetry, the space of resolvable images using all $n$ dipole patterns

$$
V=\operatorname{span}\left\{\nabla v^{(j)} \cdot \nabla v^{(i)} ; i=1, \ldots, n, \quad j=1, \ldots, n\right\}
$$

can have dimension no more than $n(n+1) / 2$. In fact, since the space of dipole current patterns is spanned by $n-1$ linearly independent dipole patterns, the dimension of $V$ can be no more than $n(n-1) / 2$.

Once $\mathbf{K}$ is computed, we can use the results of the last section to find the matrix $\tilde{\mathbf{K}}$, which is the matrix associated with the trigonometric current patterns. We can also find the determinable part of the conductivity by making use of (26).

7. Dipole patterns vs. trigonometric patterns. In this section, we describe a numerical study of the relative stability of the linearized problem for two specific sets of input current patterns: dipole patterns and trigonometric patterns. There has been some controversy over which of the two patterns actually "work best" in practical imaging systems. The results of this section suggest that in most cases, the trigonometric patterns lead to a slightly more stable problem and can provide more accurate reconstructions given a fixed condition number for the pseudo-inverse.

These experiments are based on the point-electrode model. We used the explicit representation for the gradients of the background fields $\nabla v^{(j)}$ derived in Section 6 to calculate the matrix $\mathbf{K}$ corresponding to dipole current patterns, as given by (25). From $\mathbf{K}$ we then calculated the matrix $\tilde{\mathbf{K}}=\mathbf{X} \mathbf{K X}^{T}$ corresponding to the trigonometric current patterns, where $\mathbf{X}$ is the trigonometric transformation matrix described in Section 5. To make our comparisons between current patterns "fair", we normalized the trigonometric patterns to have the same $l^{2}$ norm as the dipole patterns.

The relative stability of the two matrices $\mathbf{K}$ and $\tilde{\mathbf{K}}$ can be studied by calculating the SVDs

$$
\mathbf{K}=\mathbf{Q} \Sigma \mathbf{Q}^{T}, \quad \text { and } \tilde{\mathbf{K}}=\tilde{\mathbf{Q}} \tilde{\mathbf{\Sigma}} \tilde{\mathbf{Q}}^{T}
$$

as described in Section 4. Using the SVD, we can also calculate the pseudo-inverse and hence compare optimally reconstructed images for the two sets of current patterns.

Figure 1 shows the logarithms of the normalized singular values $\lambda_{p} / \lambda_{1}$ (sorted in decreasing order) for the matrices $\mathbf{K}$ and $\tilde{\mathbf{K}}$ describing a 16-electrode system. As we mentioned in Section 6, the dimension of the space $V$ of visible images with an $n$-electrode system (with point electrodes) can be no greater than $n(n-1) / 2$. Figure 1 
FIG. 1. Logarithm of $\lambda_{p} / \lambda_{1}$ versus $p$ for the matrices $\mathbf{K}$ and $\tilde{\mathbf{K}}$.

illustrates the rapid decay toward zero of the singular values as the index approaches $n(n-1) / 2=120$. From Figure 1 we also see that most of the relative singular values are slightly larger for the "trigonometric" matrix $\tilde{\mathbf{K}}$ than for the "dipole" $\mathbf{K}$. We made the same comparison of singular values for $n$-electrode systems with $n$ ranging from 8 to 24 -in all cases the behavior was qualitatively similar to that shown in Figure 1, with $\tilde{\mathbf{K}}$ exhibiting slightly larger relative singular values over most of the index range, and a rapid drop-off as the index $p$ approaches $n(n-1) / 2$, suggesting that higher resolution reconstruction are necessarily unstable.

Perhaps a more realistic way to compare the stability of the two problems is to apply the criterion discussed in Section 4, where we fix the condition number of the pseudo-inverse (error magnification tolerance) $M$, and construct the pseudo-inverse accordingly, i.e., include in our reconstruction only the singular vectors corresponding to singular values $\lambda_{p}$ such that $\lambda_{1} / \lambda_{p} \leq M$. In Figure 2, we have plotted the index $p$ (the number of singular vectors allowed in the reconstruction) versus the condition number of the pseudo-inverse $M$, for a 20-electrode system. Roughly speaking, this figure represents the plots of resolution versus stability for the two systems. Notice that for "moderate" values $M$ (moderately stable inversion), the number of allowed singular vectors for the trigonometric current patterns can be on the order of 20 percent more than the number of allowed vectors for dipole current patterns. This would suggest that for moderate values of $M$, a system using trigonometric current patterns will produce higher resolution images than one using dipole patterns.

Thus, the increased stability afforded by the trigonometric current patterns allows one to safely include a larger number of singular vectors in the reconstruction. The effect on the reconstruction can be very noticeable, particularly for low values of $M-$ presumably the typical case in real measurement systems. Figure 3 shows the image 
FIG. 2. Number of allowable singular vectors versus condition number $M$

of a small inclusion in the conductivity, located approximately midway between the center and the boundary of the region. Using the SVD, we calculated the "stabilized" pseudo-inverse as described in Section 4, including in the reconstruction only those singular vectors associated with singular values $\lambda_{p}$ such that $\lambda_{1} / \lambda_{p} \leq M$. In the reconstruction, we have assumed that the image $\delta \sigma$ is zero for $r>0.85$. Figure 4 shows the reconstructed images of the inclusion in Figure 3, performed with a 20electrode system and using a condition number $M=600$. In this case, the number of singular vectors allowed for the dipole system was 45 ; the trigonometric system allowed 57 (about 26 percent more). Figure 4a shows the reconstruction using dipole current patterns; Figure $4 \mathrm{~b}$ shows the reconstruction with trigonometric patterns. We note that the gray-scales are different between all three images. In fact, the absolute magnitude of the trigonometric reconstruction is almost twice that of the dipole reconstruction. Perhaps the most remarkable difference between the two reconstructions is that the dipole reconstruction was unable to resolve the location of the inclusion in the radial direction, whereas the trigonometric reconstruction, although "blurry", locates the inclusion reasonably well.

Of course, by increasing the condition number of the pseudo-inverse $M$ and hence sacrificing some stability, more accurate reconstructions can be obtained for both current patterns. In Figure 5 and Figure 6 , we compare the dipole and trigonometric reconstructions with condition numbers $M=6 \times 10^{3}$ and $M=6 \times 10^{4}$, respectively. We observe that, as expected, both reconstructions improve as $M$ increases. In addition, the difference between the dipole and trigonometric reconstructions appears to diminish for larger $M$. For $M=6 \times 10^{3}$, the dipole system allowed 61 singular vectors, compared to 71 for the trigonometric system (about 16 percent more). For $M=6 \times 10^{4}$, the dipole system allowed 79 singular vectors, with the trigonometric 
FIG. 3. Conductivity inclusion.

FIG. 4. Reconstructions with condition number $M=600$. (a.) Reconstructed image using dipole current patterns. (b.) Reconstructed image using trigonometric current patterns. 
FIG. 5. Reconstructions with condition number $M=6000$. (a.) Reconstructed image using dipole current patterns. (b.) Reconstructed image using trigonometric current patterns.

FIG. 6. Reconstructions with condition number $M=6 \times 10^{4}$. (a.) Reconstructed image using dipole current patterns. (b.) Reconstructed image using trigonometric current patterns. 
FIG. 7 . Proportion of the total number of singular vectors allowed at $M=1000$, versus number of electrodes.

system allowing 85 (about 7.6 percent more). Thus, the resolution advantage afforded by the trigonometric current patterns appears to decrease as $M$ increases. This is also reflected in Figure 1.

Given condition number in the reconstruction $M$, it is interesting to examine the number of singular vectors allowed as a percentage of the total number of singular vectors (associated with non-zero singular values), as the number of electrodes in the system is increased. This gives an indication of the amount of additional information which can be obtained by adding more electrodes to the system in the presence of fixed uncertainty in the data. Figure 7 shows graphs of the percentage of singular vectors versus number of electrodes for condition number $M=1000$. Clearly there is a downward trend. The data indicate (roughly) that the absolute number of allowable singular vectors for both dipole and trigonometric current patterns increases in direct proportion to the number of electrodes in the system. We examined the data with $M$ set at several values ranging from 500 to $10^{5}$, with results qualitatively similar to those shown in Figure 7. Again we found that the trigonometric current patterns generally allowed a higher percentage of singular vectors.

8. Discussion. In this paper we have studied the problem of characterizing the information content of the data for the linearized electrical impedance tomography problem, for imaging systems with a finite number of electrodes. We have described how to construct a pseudo-inverse operator, which gives the minimum-norm leastsquares solution to the linearized problem. We discussed how to modify the pseudosolution to take into account the inevitable uncertainty in the data. We then showed how electrical impedance imaging systems using any set of current patterns as inputs can be analyzed by studying the case where dipole current patterns are used. 
Models using different current patterns can be translated back and forth via a simple transformation matrix.

Finally, we carried out a numerical study comparing the stability and resolving power of imaging systems using dipole current patterns versus systems using trigonometric current patterns. Our numerical results suggest that trigonometric patterns usually lead to a slightly more stable system and can provide more accurate reconstructions than dipole patterns for moderate error magnification tolerances.

In our numerical investigation, we used point electrodes. Observe that the functions $\nabla v^{(j)} \cdot \nabla v^{(i)}$ [see equation (26)], which make up the image $\delta \sigma$, are singular at the boundary $r=1$ for angles $\theta_{j}, \theta_{j+1}, \theta_{i}$, and $\theta_{i+1}$. In a system with finite width electrodes, we would replace $v^{(j)}$ with $V^{(j)}$, which is a harmonic function satisfying (5b) for dipole $f$ 's. The functions $\nabla V^{(j)} \cdot \nabla v^{(i)}$ are less singular at the boundary than the functions $\nabla v^{(j)} \cdot \nabla v^{(i)}$. Therefore we conjecture that images constructed by a system with wider electrodes will be better in quality than those constructed with narrow electrodes.

Many more interesting questions remain to be addressed. Two questions in particular follow from the present work. First, our numerical study suggests that trigonometric current patterns usually lead to a more stable linearized problem. Is it possible to prove that this is the case? More generally, are there other current patterns which lead to a linearized problem which is more stable than that given by trigonometric patterns? Second, we have shown how to compute the SVD for the linearized problem. We noticed in our computations that the singular vectors are highly structured and possess remarkable symmetry properties. The question is whether it is possible to find some explicit representation for the singular vectors - at least for simple current patterns such as dipoles.

\section{REFERENCES}

[1] A. Allers And F. SAntosa, Stability and resolution analy sis of a linearized problem in electrical impedance tomography, Inverse Problems, 7 (1991), pp. 515-533.

[2] D. Barber and B. Brown, Recent developments in applied potential tomography-APT, in Information Processing in Medical Imaging, S. Bacharach, ed., Nijhoff, Amsterdam, 1986, pp. 106-121.

[3] - Progress in electrical impedance tomography, in Inverse Problems in Partial Differential Equations, D. Colton, R. Ewing, and W. Rundell, eds., SIAM, Philadelphia, 1990.

[4] D. Barber, B. Brown, and J. Jossinet, Electrical impedance tomography, Clinical Physics and Physiological Measurements, 9 (1988). Supplement A.

[5] A. Calderon, On an inverse boundary value problem, in Seminar on Numerical Analysis and its Applications, W. Meyer and M. Raupp, eds., Brazilian Mathematical Society, Rio de Janeiro, 1980, pp. 1-7.

[6] M. CHENEY AND D. IsAaCSON, Effects of measurement precision and finite number of electrodes on linear impedance imaging algorithms. preprint, 1990.

[7] M. Cheney, E. SomersaALO, AND D. IsaACSON, Existence and uniqueness for electrode models for electric current computed tomography. preprint, 1991.

[8] D. Dobson, Stability and regularity of an inverse elliptic boundary value problem. Thesis, Rice University, Department of Mathematical Sciences, 1990.

[9] D. Gisser, D. IsaACSON, AND J. Newell, Electric current computed tomography and eigenvalues, SIAM Journal of Applied Mathematics, 50 (1990), pp. 1623-1634.

[10] G. Golub and C. V. Loan, Matrix Computations, Johns Hopkins, 1983.

[11] D. IsaAcson, Distinguishability of conductivities by electric current computed tomography, IEEE Transactions in Medical Imaging, MI-5 (1986), pp. 91-95.

[12] A. Kirsch, B. Schomburg, and G. Berendt, The backus-gilbert method, Inverse Problems, 4 (1988), pp. $771-783$. 